Editorial

\title{
Joint Preservation of Knee: What Is Its Value?
}

\author{
Giuseppe Milano ${ }^{1}$ \\ ${ }^{1}$ Department of Orthopaedics, Catholic University, Service of \\ Shoulder Surgery, A. Gemelli University Hospital, Rome, Italy \\ Joints 2017;5:57-58.
}

This issue of Joints includes three articles that focus on the treatment of degenerative knee disease. Priano ${ }^{1}$ describes a study of HYADD ${ }^{8} 4$ (a hyaluronic acid derivative-based hydrogel) and its effects on symptoms and joint function in patients with symptomatic knee osteoarthritis. Adriani et $\mathrm{al}^{2}$ evaluates the safety and efficacy of autologous aspirated and purified fat tissue injected percutaneously into the knee joint for the treatment of symptomatic osteoarthritis, while Rosa et $\mathrm{al}^{3}$ reports the findings of a systematic literature review on the treatment of failed cartilage repair procedures.

Joint preservation-I refer not only to minimally invasive surgery but also to conservative treatments and damage prevention measures-is currently a very widespread and topical concept relevant not only to the knee joint. However, joint preservation strategies are often expensive and not validated for efficacy and safety, with the result that the value of these treatments is still debated.

Michael Porter, of Harvard Business School, defined patient value as outcome divided by cost. ${ }^{4}$ On the basis of his analysis, Porter formulated a concept that he termed "value-based medicine," wherein value is the best possible relationship between the optimal outcome of a treatment (i.e., restoration of the best possible health status attainable) and the expenditure incurred to achieve that result. However, if an individual patient perceives his or her health to be priceless, then cost becomes irrelevant. ${ }^{5}$ But who should foot the bill? The national healthcare system of a country plays a decisive role in the selection of treatments, given that the costs of unnecessary treatments may ultimately be borne by the community. Unfortunately, Porter's theory has a weakness that he himself recognized. In short, to calculate the value of a treatment, it is necessary to measure and evaluate its outcome, and he found that doctors, in most cases, did not know how to do this.

How should the result of a treatment be measured? There already exists a surfeit of outcome measures, some subjective and others objective, and both types carry risks of bias. With regard to patient-reported outcomes (PROs), patient expectation is known to be significantly correlated in a positive sense with subjective outcome. In other words, patients who expect more from the proposed treatment will feel that they have done better. Information, too, is significantly correlated with outcome, and this often depends not just on the communication skills of the physician and the tools he/she uses but also on the patient's ability to interpret the information given, a factor that seems to be strictly correlated with the patient's sociocultural status and education. Furthermore, patient expectations and patient information are significantly correlated with each other. It should also be added that the validity of a treatment, as can be inferred from the evidence of its efficacy reported in the literature, depends, in theory, on its integrity or fidelity, i.e., the extent to which it is implemented as intended. ${ }^{6}$ Treatment integrity is an essential condition for empirical evaluation of an intervention's efficacy, as it allows unambiguous interpretation of the results obtained. Without it, the results obtained could actually be showing a correlation with the treatment as it was administered in reality, rather than with the treatment as it was theoretically meant to have been administered. This is a hugely important concept in outcome research, particularly in areas, such as surgery and rehabilitation, where the administration of treatments may be subjected to uncontrolled variability both between and within the subjects. In practice, if a validated treatment is administered with a high level of integrity, it is probably more likely to give outcomes similar to those reported in the original efficacy study.

Even the costs are not as easy to calculate as one might imagine. For example, apart from the direct and indirect costs of a treatment, which are often underestimated, consideration should also be given to costs that are incurred as a result of doctors' decisions, ${ }^{7}$ and in this regard, how do we determine whether the treatment chosen by a doctor is the right one for a particular patient? Recently, Kibler, in an
Address for correspondence Giuseppe Milano, MD, Department of Orthopaedics, Catholic University, Service of Shoulder Surgery, A. Gemelli University Hospital, Largo Agostino Gemelli 8, 00136, Rome, Italy (e-mail: giuseppe. milano@unicatt.it).
DOI https://doi.org/ 10.1055/s-0037-1606100. ISSN 2282-4324.
Copyright ( $\odot 2017$ Georg Thieme Verlag License terms KG Stuttgart · New York 
interesting reflection on the value of treatment in orthopaedic surgery, stressed the importance of a correct diagnosis to obtain a good result, remarking that "there is a linear, almost cause-and-effect relationship between the diagnosis and the outcome." ${ }^{8}$ As explained by Rossi et al $^{9}$ in a recent editorial published in Arthroscopy that to achieve the best outcome, we need to choose the best treatment, and to choose the best treatment, we need to make an accurate diagnosis.

In outcome research, there is a lot of talk of efficacy and safety, but not enough is said about adequacy. Adequacy, basically, refers to the ability to identify the type of patient who may really benefit from a given treatment.

In the light of all these considerations, the choice of a treatment should, nowadays, be based on an individualized and shared weighing up process, involving both the physician and the patient, rather than purely on evidence-based data. Shared decision making gives both the patient and the physician the chance to decide, together, what should be done after first discussing the various options and their risks and benefits, and considering all the aspects linked to the value of the treatment, to preferences, and to the individual patient's circumstances. Naturally, all this rests on the assumption that the information needed to reach a decision is valid, i.e., that the diagnosis is correct; the integrity of the proposed treatment can be guaranteed; the expected benefits are genuinely valid in safety, effectiveness, and adequacy terms; the estimate of the direct and indirect costs is reliable; and the doctor-patient relationship is good and underpinned by proper bidirectional communication. The question to be asked, though, is: Are we truly equipped to guarantee all this?

\section{References}

1 Priano F. Early Efficacy of Intra-Articular HYADD® 4 (Hymovis ${ }^{\circledR}$ ) Injections for Symptomatic Knee Osteoarthritis. Joints 2017; 5(02):79-84

2 Adriani E, Moio M, Di Paola B, et al. Percutaneous Fat Transfer to Treat Knee Osteoarthritis Symptoms: Preliminary Results. Joints 2017;5(02):89-92

3 Rosa D, Di Donato SL, Balato G, et al. How to Manage a Failed Cartilage Repair: A Systematic Literature Review. Joints 2017; 5(02):93-106

4 Porter ME. What is value in health care? N Engl J Med 2010;363 (26):2477-2481

5 Rossi MJ, Brand JC, Provencher MT, Lubowitz JH. Shoulder arthroscopy complication and readmission rates: impact on value. Arthroscopy 2017;33(01):4-5

6 Vermilyea BB, Barlow DH, O'Brien GT. The importance of assessing treatment integrity: an example in the anxiety disorders. J Behav Assess 1984;6(01):1-11

7 Srikumaran U, Hannan C, Higgins L, Warner J. Value and health care policy ramifications of rotator cuff repair. Symposium Techn Orthopaed 2016;31:120-126

8 Kibler WB. Value on the front end: making the effective diagnosis for optimal treatment. Arthroscopy 2017;33(02): 493-495

9 Rossi MJ, Lubowitz JH, Brand JC, Provencher MT. Making the right treatment decision requires consideration of utility and reconsideration of value. Arthroscopy 2017;33(02): 239-241 